\title{
The Veterans Choice Act and Dual Health System Use
}

\author{
Walid F. Gellad, MD, MPH', \\ 'Division of General Internal Medicine, Department of Medicine, University of Pittsburgh School of Medicine, Pittsburgh, PA, USA; ${ }^{2}$ Center for Health \\ Equity Research and Promotion, VA Pittsburgh Healthcare System, Pittsburgh, PA, USA.
}

$J$ Gen Intern Med 31(2):153-4

DOI: $10.1007 / \mathrm{s} 11606-015-3492-2$

(c) Society of General Internal Medicine 2015

$\mathrm{T}$ he Veterans Access, Choice and Accountability Act (Choice Act), formally enacted on August 7, 2014, arose largely in response to outrage about delays in veteran access to health care and falsification of wait time records by Veterans Administration (VA) employees. ${ }^{1}$ The law includes a number of provisions to improve care for veterans, including increasing health care staffing and residency training positions, streamlining employee disciplinary procedures, improving treatment of military sexual trauma, and establishing a Presidential Commission on Care to evaluate access to VA health care. The most well-known, and perhaps controversial, provision is the Choice Program, which established a mechanism for eligible veterans to receive care outside the VA for 3 years. Veterans unable to schedule an appointment within 30 days of their preferred date or who live more than 40 miles from a VA facility are now eligible to receive care from non-VA providers. However, many of these veterans will also continue to use VA facilities and services, and this 'dual use' of health care systems may lead to care fragmentation and its ensuing adverse consequences. This commentary describes the problems of dual use and care fragmentation, the complexity of the Choice Program, and some ideas for ensuring its safe and effective implementation.

The challenges surrounding the implementation of the Choice Program have been monumental. In the 90 days between passage of the law and the start of the program on November 5, 2014, the VA developed a system for identifying and reaching out to eligible veterans, tracking Choice Program visits, and paying for services through the program. As of April 2015, the VA had coordinated more than 45,000 Choice Program medical appointments and dispensed more than 3,200 Choice prescriptions. ${ }^{2}$ However, this implementation has received tremendous public criticism because of the relatively low uptake of services compared to the perceived need for immediate care. In response, in May of 2015, the U.S. Senate unanimously voted to amend the Choice Act, expanding access to veterans living within 40 miles of a VA facility if it does not offer them the needed medical care. The

Published online August 20, 2015 measure, however, has not passed the U.S. House of Representatives.

The Choice Program is not the first time that an expansion of non-VA care for veterans has increased the complexity or fragmentation of care. In 2006, the rollout of Medicare Part D prescription drug coverage gave millions of VA patients the opportunity to obtain prescription drugs through the private health care system. Despite almost 10 years having elapsed since Part D's implementation, however, and the concerns related to dual use, there are limited data on its impact on care fragmentation among veterans. In 2014, the Affordable Care Act (ACA) again expanded access for veterans to non-VA care through both the private insurance marketplace and Medicaid. Similar concerns have been raised about increased care fragmentation with dual use as a consequence of the ACA, although the full impact will not be known for some time. ${ }^{3}$

Even before these insurance expansions, veterans with Medicare coverage were known to receive care through both Medicare and VA. Prior work has documented convincing evidence that dual use of Medicare and VA services comes with inherent risks of care fragmentation and duplication of services, including, for example, higher rates of hospitalization for ambulatory care-sensitive conditions, higher cost and worse outcomes in cancer, and higher rates of rehospitalization. ${ }^{3,}{ }^{4}$ Despite the best efforts, care received outside the VA cannot be seamlessly integrated into the sophisticated electronic medical record (EMR) and decision support systems that unify the VA. Physicians outside the VA do not have access to the VA's EMR and ordering systems, leading to risks of duplication of services, errors, and inefficient care.

These risks of care fragmentation with dual use extend to the Choice Program, especially given its complexity. As an example, consider how prescription drugs are dispensed through Choice. Under the Program, non-VA providers must prescribe within the same formulary rules that guide VA providers. Choice providers do not have access to the electronic ordering with decision support that helps VA providers prescribe within formulary guidelines. In 2003, when VA temporarily implemented the Transitional Pharmacy Benefit for patients waiting more than 30 days for their initial primary care appointment, roughly $40 \%$ of prescriptions received from non-VA providers were non-formulary, substantially increasing the workload and costs for VA pharmacy. ${ }^{5}$ Each Choice prescription must be faxed, mailed, or delivered in person to a VA facility to be processed; verbal and telephone prescriptions 
are not accepted for safety reasons, and electronic prescribing is impossible. Once the prescription arrives at the VA pharmacy, a pharmacist adjudicates the prescription similarly to VA medications; if the medication is non-formulary, the pharmacist communicates with the ordering provider, which cannot be done electronically and transparently as is current practice in VA facilities. In cases of emergent prescriptions or discharge medications after a hospitalization, these prescriptions must be obtained from non-VA pharmacies. The system was clearly not optimized for efficient, coordinated, or safe use - it was optimized to ensure immediate access, as the law demanded.

Given the implementation challenges and inherent risks when care is fragmented, what can be done to ensure the safe and effective receipt of care in the Choice Act era? First, we should make sure that the risks of care fragmentation are brought to the fore in discussions about the Choice Program. While it is critical to ensure that veterans have access to the care they need, it is also essential to consider the associated risks of care fragmentation. As a first step, the Presidential Commission on Care established through the Choice Act should expand its current mandate of evaluating access to care to include an assessment of fragmentation and quality of care. Acknowledging the inherent risks in sending VA users to receive care outside the VA will allow for a more reasoned conversation about the potential risks and benefits of privatizing VA services. This acknowledgment is especially important given the Commission's second mandate, which is to examine how best to organize the VA and deliver care to veterans for the next 20 years.

Second, an early, robust, and informed evaluation of care under the Choice Program is needed in order to understand the nature and extent of any care fragmentation. With the right data, this evaluation can easily be done in concert with multiple ongoing evaluations of prior insurance expansions that have led to increasing dual use among veterans. However, if these evaluations are rushed to fit political timelines, then veterans are not being served in the way they should be. If the Choice Program increases access without leading to unacceptably frequent duplicative testing, dangerous drug-drug interactions, unsustainable costs, or poorer quality, the program may then be considered a success. Examining access alone, however, without considering or accurately measuring these unintended consequences is a disservice to veterans.

Third, the VA must ensure that medical records from Choice services, whether physician visits, imaging tests, or surgeries, are easily accessible to VA physicians who may still be treating those veterans, or who will treat them in a few years when authorization for the program ends. The third-party administrators who are managing the Choice program for VA are obliged to collect documentation by Choice providers. However, these documents will be scanned into the VA EMR in a section that is separate from other related pieces of information - for example, laboratory tests performed under Choice will not be found in the same section as tests performed in a VA facility, and instead may be buried in pages of scanned material. This same is true for surgical reports and office notes. The VA must develop a more effective way to integrate non-VA data for VA providers, perhaps by requiring laboratory, imaging, and provider notes from outside VA to be seamlessly embedded with VA data in the relevant sections of the EMR. Furthermore, VA must also consider the optimal strategy for sharing VA data with non-VA providers. The VA 'Blue Button' tool already allows VA medical information to be downloaded and printed by veterans who are users of the VA's personal health record, and VA is pilot testing health information exchange systems with non-VA providers in certain areas. The challenge, again, is ensuring that the information is in a format that can be useful to the non-VA provider at the point of care. Ultimately, if dual health care system use is to become the norm within VA, improving medical data sharing has to become a priority.

Veterans deserve timely health care, and in some cases they have not received that care within the VA system. However, the rush to improve access does not mean that quality can be ignored, and care fragmentation is not compatible with the highest-quality care. Veterans being seen in two different health systems, with different electronic records and different policies and procedures, face risks from dual-system health care use that should not be ignored. Moving forward, these risks must be acknowledged and addressed alongside the benefits of improving access and instituting 'Choice.'

Conflict of Interest Statement: The author is an employee of the Department of Veterans Affairs and receives grant funding from the VA to study dual health system use by veterans.

Funding: Dr. Gellad is supported by VA HSR\&D Merit Review Award IO1 HX001765.

\section{Financial Disclosures: None}

Disclaimer: The views expressed herein are solely those of the author and do not necessarily represent the views of the Department of Veterans Affairs or the. US. Government.

Corresponding Author: Walid F. Gellad, MD, MPH; Center for Health Equity Research and Promotion, VA Pittsburgh Healthcare System, University Drive, 151C, Pittsburgh, PA 15240, USA (e-mail: walid.gellad@va.gov).

\section{REFERENCES}

1. Chokshi DA. Improving Health Care for Veterans-A Watershed Moment for the VA. N Engl J Med. 2014;371(4):297-9.

2. US Department of Veterans Affairs. Progress Report: Veterans Access, Choice, and Accountability Act 2014. [2015 March 24]. (http://www.va. gov/opa/choiceact/documents/FactSheets/Progress-Report-March-2015Fact-Sheet.pdf). Accessed July 28, 2015.

3. Kizer KW. Veterans and the Affordable Care Act. JAMA. 2012;307(8):78990.

4. Pizer SD. What are the Quality Consequences of Medicare-VA Dual Use? [2013 May]. VA HSR\&D Forum. (http://www.hsrd.research.va.gov/publications/ internal/forum04_13.pdf). Accessed July 28, 2015.

5. US Department of Veterans Affairs. Statement of Dr. Jonathan Perlin, Deputy Under Secretary for Health. [2004 March 30]. (https://www.va.gov/ OCA/testimony/hvac/sh/040330JP.asp). Accessed July 28, 2015. 